\title{
ORTHONORMAL SETS WITH NON-NEGATIVE DIRICHLET KERNELS. II
}

\author{
BY \\ J. J. PRICE(1)
}

1. Introduction. Recently the author has shown [2] that an orthonormal set of functions whose associated Dirichlet kernels are non-negative must be a system of step functions similar in structure to the classical Haar functions. The present paper discusses systems in which infinitely many of the kernels, but not necessarily all, are non-negative. It is shown that such systems also must be composed of step functions of a special type. As an application, a characterization of the Walsh functions is given in $\$ 4$.

2. Definitions and preliminaries. It will be assumed throughout that $\mu$ is a totally finite measure on a space $S$ normalized so that $\mu(S)=1$. All sets mentioned will be subsets of $S$. All functions will be real-valued, bounded, and $\mu$-measurable. For the sake of brevity "almost everywhere" qualifications will be omitted. For example, two functions which differ only on a set of measure zero will be tacitly identified and "essential supremum" will be replaced by "supremum."

A partition of $S$ is a finite collection $P$ of disjoint subsets of $S$ whose union is $S$. Given partitions $P_{1}$ and $P_{2}, P_{1}>P_{2}$ if each element of $P_{2}$ is contained in an element of $P_{1}$ and if $P_{1}$ and $P_{2}$ are not identical. A function which is constant on each element of a partition $P$ will be called a step function $(P)$.

Lemma 1. Let $f(t)$ be a function defined on a set $T$ of measure $\mu_{T}$. If

$$
\begin{gathered}
-M_{2} \leqq f(t) \leqq M_{1}, \\
\int_{T} f(t) d \mu(t)=I,
\end{gathered}
$$$$
t \in T,-M_{2}<M_{1}
$$

then

$$
\int_{T} f^{2}(t) d \mu(t) \leqq M_{1} M_{2} \mu_{T}+\left(M_{1}-M_{2}\right) I
$$

Equality holds in (c) if and only if

$$
f(t) \equiv \begin{cases}M_{1}, & t \in T_{1}, \\ -M_{2}, & t \in T_{2},\end{cases}
$$

where $\left\{T_{1}, T_{2}\right\}$ is a partition of $T$ such that

Presented to the Society September 3, 1959; received by the editors August 4, 1960.

(1) This work was supported by the National Science Foundation under research grant NSF G-8818. 
(e)

$$
\mu\left(T_{1}\right)=\frac{M_{2 \mu_{T}}+I}{M_{1}+M_{2}}, \quad \mu\left(T_{2}\right)=\frac{M_{1} \mu_{T}-I}{M_{1}+M_{2}} .
$$

The proof of the special case $I=0$ is easy and is given in [2]. We shall omit it here. If $I \neq 0$, the lemma is obtained immediately by applying the special case to $g(t)=f(t)-I \mu_{T}^{-1}$.

Lemмa 2. Let $f(t)$ be defined on a set $T$ of measure $\mu_{T}$. Suppose

$$
\begin{array}{lrl}
-M_{2} \leqq f(t) \leqq M_{1}, & t \in T,-M_{2}<M_{1}, \\
\int_{T} f(t) d \mu(t)=0, & \\
\int_{T} f^{2}(t) d \mu(t) \geqq\left(M_{1}-a / 2\right) M_{2} \mu_{T}, & 0<a<M_{1} .
\end{array}
$$

Then, if $\sigma_{a}=\left\{t \mid f(t) \geqq M_{1}-a\right\}$,

$$
\mu\left(\sigma_{a}\right) \geqq \frac{1}{2} \frac{M_{2 \mu} T}{M_{1}+M_{2}} .
$$

Proof. On $\sigma_{a},-\left(a-M_{1}\right) \leqq f(t) \leqq M_{1}$, and on the complement $\sigma_{a}^{*},-M_{2}$ $\leqq f(t)<M_{1}-a$. Let $I$ and $-I$ denote the integrals of $f(t)$ over $\sigma_{a}$ and $\sigma_{a}^{*}$ and let $\mu_{a}=\mu\left(\sigma_{a}\right)$. Applying Lemma 1 twice,

$$
\begin{aligned}
& \int_{\sigma_{a}} f^{2}(t) d \mu(t) \leqq M_{1}\left(a-M_{1}\right) \mu_{a}+\left(2 M_{1}-a\right) I \\
& \int_{\sigma_{a}^{*}} f^{2}(t) d \mu(t) \leqq\left(M_{1}-a\right) M_{2}\left(\mu_{T}-\mu_{a}\right)+\left(M_{1}-a-M_{2}\right)(-I) .
\end{aligned}
$$

Adding, and using assumption (c),

$$
\begin{aligned}
\left(M_{1}-\frac{a}{2}\right) M_{2 \mu T} & \leqq \int_{T} f^{2}(t) d \mu(t) \\
& \leqq\left(M_{1}-a\right) M_{2 \mu_{T}}+\left(M_{1}+M_{2}\right)\left(a-M_{1}\right) \mu_{a}+\left(M_{1}+M_{2}\right) I .
\end{aligned}
$$

Subtracting $\left(M_{1}-a\right) M_{2} \mu_{T}$,

$$
\frac{a}{2} M_{2 \mu_{T}} \leqq\left(M_{1}+M_{2}\right)\left[a \mu_{a}+\left(I-M_{1} \mu_{a}\right)\right] .
$$

Since $0 \leqq I \leqq M_{1} \mu_{a}$, and $M_{1}+M_{2}>0$ (because of (a) and (b)),

$$
\frac{a}{2} M_{2 \mu_{T}} \leqq\left(M_{1}+M_{2}\right) a \mu_{a}
$$


or,

$$
\frac{1}{2} \frac{M_{2} \mu_{T}}{M_{1}+M_{2}} \leqq \mu_{a} .
$$

3. Main theorem. With any orthonormal set $\left\{f_{j}(s)\right\}_{j=0}^{\infty}$ in $L^{2}(S, \mu)$ are associated the Dirichlet kernels

$$
D_{n}(s, t)=\sum_{j=0}^{n-1} f_{j}(s) f_{j}(t)
$$

The following theorem gives the structure of an orthonormal set when infinitely many of these kernels are non-negative.

THEOREM 1. Let $\mathcal{F}=\left\{f_{j}(s)\right\}_{j=0}^{\infty}$ be an orthonormal set in $L^{2}(S, \mu)$ with $f_{0}(s)$ $\equiv 1$. Suppose there exists a sequence of integers $1=n_{0}<n_{1}<n_{2}<\cdots$ such that $D_{n_{r}}(s, t) \geqq 0, r \geqq 0$. Then $\mathcal{F}$ is a system of step functions of the following type. There exists a sequence $P_{0}>P_{1}>P_{2}>\cdots$ of partitions of $S, P_{r}=\left\{S_{r, i}\right\}_{i=1}^{n_{r}}$, such that if $0 \leqq j<n_{r}$, then $f_{j}(s)$ is a step function $\left(P_{r}\right), r \geqq 0$. Furthermore,

$$
D_{n_{r}}(s, t)=\left\{\begin{array}{lr}
p_{r, i}, & (s, t) \in S_{r, i}^{2}, 1 \leqq i \leqq n_{r}, \\
0, & \text { otherwise, }
\end{array}\right.
$$

where

$$
p_{r, i}=\frac{1}{\mu\left(S_{r, i}\right)}
$$

Proof. It will be shown by induction that for each $k \geqq 0$, there exists a sequence of partitions $P_{0}>P_{1}>P_{2}>\cdots>P_{k}$ of $S$ such that, if $0 \leqq r \leqq k$,

(i) $f_{j}(s)$ is a step function $\left(P_{r}\right)$ when $0 \leqq j<n_{r}$,

(ii) $P_{r}$ has exactly $n_{r}$ elements, $\left\{S_{r, i}\right\}_{i=1}^{n_{r}}$,

(iii) (2) and (3) hold.

For $k=0$, the situation is trivial. Since $f_{0}(s) \equiv 1, D_{n_{0}}(s, t) \equiv 1$. Taking $P_{0}$ to be the identity partition $\{S\}=\left\{S_{0,1}\right\}$ all assertions are obviously true. Assuming the proposition is true for $k$, we must construct a partition $P_{k+1}<P_{k}$ and show (i), (ii), (iii) can be extended to $P_{k+1}$.

For simplicity, let $D_{n_{r}}(s, t)=\Delta_{r}(s, t)$. Define

$$
F_{k}(s, t)=\Delta_{k+1}(s, t)-\Delta_{k}(s, t)=\sum_{j=n_{k}}^{n k+1-1} f_{j}(s) f_{j}(t) .
$$

We shall show that $F_{k}(s, t)=0$ wherever $\Delta_{k}(s, t)=0$, namely outside the set

$$
\bigcup_{i=1}^{n k} S_{k, i}^{2}
$$

Let $t_{0}$ be arbitrary but fixed, say $t_{0} \in S_{k, j}$. 


$$
F_{k}\left(s, t_{0}\right) \geqq 0,
$$

where the asterisk denotes complement. This follows from (4) since $\Delta_{k+1}(s, t)$ $\geqq 0$ and $\Delta_{k}\left(s, t_{0}\right) \equiv 0, s \in S_{k, j}^{*}$, by the induction hypothesis (2). Also by (2), $p_{k, j}^{-1} \Delta_{k}\left(s, t_{0}\right)$ is the characteristic function of $S_{k, j}$. Hence

$$
\begin{aligned}
\int_{S_{k, j}^{*}} F_{k}\left(s, t_{0}\right) d \mu(s) & =\int_{S}-\int_{S_{k, j}} \\
& =\int_{S} F_{k}\left(s, t_{0}\right) d \mu(s)-\frac{1}{p_{k, j}} \int_{S} \Delta_{k}\left(s, t_{0}\right) F_{k}\left(s, t_{0}\right) d \mu(s) .
\end{aligned}
$$

Both integrals on the right side of (6) vanish because $F_{k}\left(s, t_{0}\right)$ is orthogonal to $f_{0}(s) \equiv 1$ and to $\Delta_{k}\left(s, t_{0}\right)$. Therefore,

$$
\int_{S_{k, j}^{*}} F_{k}\left(s, t_{0}\right) d \mu(s)=0 .
$$

It follows from (5) and (7) that $F_{k}\left(s, t_{0}\right) \equiv 0$ on $S_{\boldsymbol{k}, y}^{*}$. Since $t_{0}$ was arbitrary, $F_{k}(s, t) \equiv 0$ outside the sets $S_{k, i}^{2}, 1 \leqq i \leqq n_{k}$.

We now begin the construction of the partition $P_{k+1}$. This will be done by partitioning separately each of the sets $S_{k, i}, 1 \leqq i \leqq n_{k}$.

The quantity

$$
F_{k}(t, t)=\sum_{j=n_{k}}^{n k+1-1} f_{j}^{2}(t)
$$

does not vanish identically on $S$. It is no loss of generality to assume $\sup _{s_{k, 1}} F_{k}(t, t)=m_{1}>0$.

Let us carry out the partitioning of $S_{k, 1}$ in detail. Henceforth, all points mentioned, unless otherwise stated, will be understood to belong to $S_{k, 1}$.

The first step is to show that $F_{k}(t, t) \equiv m_{1}$ on a set $T_{1}$ of positive measure. Choose a sequence $\left\{t_{n}\right\}_{n=1}^{\infty}$ such that $F_{k}\left(t_{n}, t_{n}\right) \geqq m_{1}(1-1 / 2 n)$. Then

$$
\begin{aligned}
& -p_{k, 1} \leqq F_{k}\left(s, t_{n}\right) \leqq m_{1}, \\
& \int_{S_{k, 1}} F_{k}\left(s, t_{n}\right) d \mu(s)=0, \\
& \int_{S_{k, 1}} F_{k}^{2}\left(s, t_{n}\right) d \mu(s) \geqq m_{1}\left(1-\frac{1}{2 n}\right) .
\end{aligned}
$$

The upper bound in (8a) is a consequence of the Schwarz inequality,

$$
F_{k}(s, t)=\sum_{j=n_{k}}^{n_{k+1}-1} f_{j}(s) f_{j}(t) \leqq\left(F_{k}(s, s) F_{k}(t, t)\right)^{1 / 2}
$$


The lower bound in (8a) follows from (4) and the facts that $\Delta_{k}\left(s, t_{n}\right) \leqq p_{k, 1}$ by the induction hypothesis and that $\Delta_{k+1}\left(s, t_{n}\right) \geqq 0$. (8b) is obtained from (7) since $F_{k}\left(s, t_{n}\right)$ is orthogonal (over $S$ ) to $f_{0}(s) \equiv 1$. Finally, using orthonormality, one obtains the general identity

$$
\int_{S_{k=1}} F_{k}\left(s, t_{\alpha}\right) F_{k}\left(s, t_{\beta}\right) d \mu(s)=F_{k}\left(t_{\alpha}, t_{\beta}\right) .
$$

Putting $t_{\alpha}=t_{\beta}=t_{n}$ yields $(8 \mathrm{c})$.

Now $(8 \mathrm{a}, \mathrm{b}, \mathrm{c})$ are precisely the conditions needed to apply Lemma 2 to $F_{k}\left(s, t_{n}\right)$ with $T=S_{k, 1}, \mu_{T}=p_{k, 1}^{-1}, M_{1}=m_{1}, M_{2}=p_{k, 1}$ and $a=m_{1} / n$. We obtain

$$
\mu\left(\sigma_{n}\right) \geqq \frac{1}{2} \frac{1}{m_{1}+p_{k, 1}}=c_{1}
$$

where $\sigma_{n}=\left\{s \mid F_{k}\left(s, t_{n}\right) \geqq m_{1}(1-1 / n)\right\}$. Now let $\tau_{n}=\left\{s \mid F_{k}(s, s) \geqq m_{1}(1-1 / n)^{2}\right\}$. Then $\sigma_{n} \subset \tau_{n}$. For, by (9), if $s \in \sigma_{n}$

$$
F_{k}(s, s) m_{1} \geqq F_{k}(s, s) F_{k}\left(t_{n}, t_{n}\right) \geqq F_{k}^{2}\left(s, t_{n}\right) \geqq m_{1}^{2}(1-1 / n)^{2} .
$$

Consequently $\mu\left(\tau_{n}\right) \geqq \mu\left(\sigma_{n}\right) \geqq c_{1}>0, \quad n \geqq 1$. If $T_{1}=\left\{s \mid F_{k}(s, s)=m_{1}\right\}$ then $T_{1}=\bigcap_{n=1}^{\infty} \tau_{n}$. But $\tau_{1} \supset \tau_{2} \supset \tau_{3} \supset \ldots$. Therefore, $\mu\left(T_{1}\right) \geqq c_{1}>0$.

We may now obtain some precise information about $F_{k}(s, t)$ as follows. Using $(8 \mathrm{a}, \mathrm{b})$, we may apply Lemma 1 to $F_{k}(s, t)$ with $t$ fixed and obtain

$$
\int_{S_{k, 1}} F_{k}^{2}(s, t) d \mu(s) \leqq m_{1} .
$$

On the other hand, if $t \in T_{1}$, we have from (10)

$$
\int_{S_{k, 1}} F^{2}(s, t) d \mu(s)=F_{k}(t, t)=m_{1} .
$$

Thus, $F_{k}(s, t)$ is an extremal function in the sense of Lemma 1 when $t \in T_{1}$. Therefore, for each $t \in T_{1}$, there exists a set $V(t)$ such that

$$
F_{k}(s, t) \equiv\left\{\begin{array}{l}
m_{1}, \\
-p_{k, 1},
\end{array}\right.
$$
otherwise,

where

$$
\mu(V(t))=\frac{1}{m_{1}+p_{k, 1}} .
$$

We are going to show that there are only a finite number of distinct sets $V(t)$, that these form a partition $P\left(T_{1}\right)$ of $T_{1}$, and that the functions $f_{j}(s)$, $0 \leqq j<n_{k+1}$, are step functions $\left(P\left(T_{1}\right)\right)$.

Let $t \in T_{1}$ and $s \in V(t)$. From (9) and (11), 


$$
m_{1}=F_{k}(s, t) \leqq\left(F_{k}(s, s) F_{k}(t, t)\right)^{1 / 2} \leqq m_{1} .
$$

Hence, $F_{k}(s, s)=m_{1}$ which shows that $V(t) \subset T_{1}$. Since $t \in V(t), T_{1}$ $=U_{t \in T 1} V(t)$.

Also from (13),

$$
\sum_{j=n_{k}}^{n_{k+1}-1} f_{j}(s) f_{j}(t)=\left(\sum_{j=n_{k}}^{n_{k}+1-1} f_{j}^{2}(s) \sum_{j=n_{k}}^{n_{k+1-1}} f_{j}^{2}(t)\right)^{1 / 2} .
$$

Consequently, for $t$ fixed, there exists a proportionality factor $\lambda(s)$ such that $f_{j}(s)=\lambda(s) f_{j}(t)$ for all $s \in V(t), n_{k} \leqq j<n_{k+1}$. Therefore, $F_{k}(s, t)=\lambda(s) F_{k}(t, t)$. But $F_{k}(s, t) \equiv F_{k}(t, t)=m_{1}, s \in V(t)$, by (11). It follows that $\lambda(s) \equiv 1$ on $V(t)$. Therefore, the functions $f_{j}(s), n_{k} \leqq j<n_{k+1}$, are constant on $V(t)$. (By the induction hypothesis, this is also true for $0 \leqq j<n_{k}$ since $f_{j}(s)$ is then constant on the superset $S_{k, 1}$.)

It is now clear that $F_{k}(s, \tau) \equiv F_{k}(t, t)=m_{1},(s, \tau) \in V^{2}(t)$. Hence, if $t^{\prime} \in V(t)$, $F_{k}\left(s, t^{\prime}\right) \equiv m_{1}$ for $s \in V(t)$. Since $V\left(t^{\prime}\right)$ is that set of measure $\left(m_{1}+p_{k, 1}\right)^{-1}$ on which $F_{k}\left(s, t^{\prime}\right) \equiv m_{1}$, we have that $V\left(t^{\prime}\right)=V(t)$. It follows that if $t_{1}, t_{2} \in T_{1}$, then $V\left(t_{1}\right)$ and $V\left(t_{2}\right)$ are either disjoint or identical. In other words, the sets $V(t)$ form a partition $P\left(T_{1}\right)$ of $T_{1}$. Since they all have the same positive measure and $\mu\left(T_{1}\right)$ is finite, $P\left(T_{1}\right)$ is a finite partition. Let us call the elements of this partition $S_{k+1, i}, 1 \leqq i \leqq q_{1}$. Define $p_{k+1, i}=\mu\left(S_{k+1, i}\right)^{-1}=m_{1}+p_{k, 1}$.

To summarize, we have established the following facts. There exists a set of positive measure $T_{1}$ on which $F_{k}(t, t) \equiv m_{1}$. There is a partition $P\left(T_{1}\right)$ $=\left\{S_{k+1, i}\right\}_{i=1}^{q_{1}}$ of $T_{1}$ into sets of equal measure such that $f_{j}(s)$ is a step function $\left(P\left(T_{1}\right)\right)$ for $0 \leqq j<n_{k+1}$. Furthermore,

$$
F_{k}(s, t) \equiv\left\{\begin{array}{lr}
m_{1}=p_{k+1, i}-p_{k, 1}, & (s, t) \in S_{k+1, i}^{2}, 1 \leqq i \leqq q_{1}, \\
-p_{k, 1}, & \text { elsewhere on } S_{k, 1} \times T_{1} \cup T_{1} \times S_{k, 1} .
\end{array}\right.
$$

Now $\Delta_{k+1}(s, t)=\Delta_{k}(s, t)+F_{k}(s, t)$. We obtain from (14) and the induction hypothesis (2) that

$$
\Delta_{k+1}(s, t) \equiv\left\{\begin{array}{lr}
p_{k+1, i}, & (s, t) \in S_{k+1, i}^{2}, 1 \leqq i \leqq q_{1}, \\
0, & \text { elsewhere on } S_{k, 1} \times T_{1} \cup T_{1} \times S_{k, 1},
\end{array}\right.
$$

where

$$
p_{k+1, i}=\frac{1}{\mu\left(S_{k+1, i}\right)} .
$$

(15) and (16) represent a partial extension of (2) and (3) to the case $r=k+1$. If $T_{1}=S_{k, 1}$ we have the desired partition of $S_{k, 1}$.

Suppose then, that $T_{1}$ is a proper subset of $S_{k, 1}$. Let $m_{2}=\sup F_{k}(t, t)$, $t \in T_{1}^{*} . m_{2}$ must be positive. For otherwise $f_{j}(t) \equiv 0$ on $T_{1}^{*}, n_{k} \leqq j<n_{k+1}$. Hence $F_{k}(s, t) \equiv 0$ outside of $T_{1}^{2}$ which contradicts (14). (Note that $m_{2} \leqq m_{1}$.) 
Using the above arguments, we can easily establish the following. There exists a set $T_{2} \subset T_{1}^{*}, \mu\left(T_{2}\right) \geqq c_{2}=(1 / 2)\left(m_{2}+p_{k, 1}\right)^{-1}$, such that $F_{k}(t, t) \equiv m_{2}$ on $T_{2}$. There is a partition $P\left(T_{2}\right)=\left\{S_{k+1, i}\right\}_{i=q_{1}+1}^{q_{2}}$ of $T_{2}$ into sets of equal measure such that the functions $f_{j}(s), 0 \leqq j<n_{k+1}$ are step functions $\left(P\left(T_{2}\right)\right)$ and the analogues of (15) and (16) hold.

Continuing in this way, we obtain sets $T_{1}, T_{2}, \cdots$ such that $F_{k}(t, t)$ $\equiv m_{\nu}>0$ on $T_{\nu}$ and $\mu\left(T_{\nu}\right) \geqq c_{\nu}=2^{-1}\left(m_{\nu}+p_{k, 1}\right)^{-1}$. The process terminates after a finite number of steps since $\mu\left(T_{\nu}\right) \geqq 2^{-1}\left(m_{\nu}+p_{k, 1}\right)^{-1} \geqq 2^{-1}\left(m_{1}+p_{k, 1}\right)^{-1}=c_{1}$ while $\mu\left(S_{k, 1}\right)$ is finite. The sets $T_{\nu}$ form a finite partition of $S_{k, 1}$. Each of these is partitioned in the same way as $T_{1}$. The result is a partition of $S_{k, 1}$ possessing all the required properties.

(We now drop the convention that all points named belong to $S_{k, 1 .}$ ) Each of the sets $S_{k, i}, 2 \leqq i \leqq n_{k}$, can be partitioned in the same way provided $\sup _{S_{k, i}} F_{k}(t, t)>0$. If $\sup _{S_{k, i}} F_{k}(t, t)=0$, then $f_{j}(s) \equiv 0$ on $S_{k, i}, n_{k} \leqq j<n_{k+1}$. In this case $\Delta_{k+1}(s, t) \equiv \Delta_{k}(s, t)$ on $S_{k, i}^{2}$ and (2) and (3) trivially carry over if we take the identity partition $\left\{S_{k, i}\right\}$.

Combining these partitions we obtain a partition $P_{k+1}=\left\{S_{k+1, i}\right\}_{i=1}^{N}$ of $S$ such that $P_{k+1}<P_{k}, f_{j}(s)$ is a step function $\left(P_{k+1}\right)$ if $0 \leqq j<n_{k+1}$, and

$$
\Delta_{k+1}(s, t) \equiv\left\{\begin{array}{lr}
p_{k+1, i}, & (s, t) \in S_{k+1, i}^{2}, 1 \leqq i \leqq N \\
0, & \text { otherwise }
\end{array}\right.
$$

where

$$
p_{k+1, i}=\frac{1}{\mu\left(S_{k+1, i}\right)}
$$

To complete the induction, it remains only to show that $N=n_{k+1}$. By orthonormality,

$$
\int_{S}\left(\int_{S} \Delta_{k+1}^{2}(s, t) d \mu(s)\right) d \mu(t)=n_{k+1} .
$$

On the other hand this integral is easily computed as a double integral from (17) and (18). Its value is

$$
\sum_{i=1}^{N} p_{k+1, i}^{2} \mu\left(S_{k+1, i}\right)^{2}=\sum_{i=1}^{N} 1=N
$$

Hence, $N=n_{k+1}$.

It is worth noting the following facts, all of which follow directly from the proof of Theorem 1 but are not given in the statement of the theorem. $P_{k+1}$ is obtained from $P_{k}$ by partitioning each set $S_{k, i}$ into two or more subsets unless $F_{k}(t, t) \equiv 0$ on $S_{k, i}$. In particular, if $F_{k}(t, t)>0$ for all $t$ and $n_{k+1}$ $=2 n_{k}$, then each element of $P_{k}$ splits into exactly two parts. If $n_{k+1}<2 n_{k}$, then 
$F_{k}(t, t) \equiv 0$ on a set of positive measure. Finally we observe that if $F_{k}(t, t)$ is constant on $S_{k, i}$, then $S_{k, i}$ is partitioned into sets of equal measure.

4. An application to Walsh functions. In this section the unit interval $\{x \mid 0 \leqq x<1\}$ will be denoted by $I$, the dyadic interval

$$
\left\{x \mid r \cdot 2^{-k} \leqq x<(r+1) 2^{-k}\right\} \text { by } I(r, k),
$$

and the dyadic partition $\{I(r, k)\}_{r=0}^{2^{k-1}}$ of $I$ by $J_{k}$.

The Walsh functions $\left({ }^{2}\right)$ are step functions related to the sequence of partitions $J_{0}>J_{1}>J_{2}>\cdots$ in the sense of Theorem 1. This suggests a characterization of the Walsh system by its Dirichlet kernels.

THEOREM 2. Let $\mathcal{F}=\left\{f_{n}(x)\right\}_{n=0}^{\infty}$ be an orthonormal set on I with the following properties.

(a) $f_{0}(x) \equiv 1$.

(b) $D_{2^{k}}(x, y) \geqq 0, k \geqq 0$.

(c) For each $n \geqq 0$, there is a partition $Q_{n}=\left\{Q_{n, j}\right\}_{j=0}^{n}$ of $I$ into $n+1$ subintervals on which $f_{n}(x)$ is alternately non-negative and nonpositive. $\left(f_{n}(x)\right.$ is non-negative on the sub-interval containing 0.$)$

(d) For each $n, \sup _{Q_{n, j}}\left|f_{n}(x)\right|$ is independent of $j$.

Then $\mathcal{F}$ is the set of Walsh functions.

Proof. By Theorem 1, assumptions (a) and (b) imply that $\mathcal{F}$ is a system of step functions relative to a sequence of partitions $P_{0}>P_{1}>P_{2}>\cdots$ of $I, P_{k}$ having $2^{k}$ elements. It follows from (d) and the fact that $f_{n}(x)$ is normalized that $\left|f_{n}(x)\right| \equiv 1$. Consequently,

$$
F_{k}(x, x) \equiv \sum_{n=2^{k}}^{2 k+1-1} f_{n}^{2}(x) \equiv 2^{k} .
$$

By the remarks following Theorem 1, $P_{k+1}$ arises by splitting each element of $P_{k}$ into two subsets of equal measure. These must be intervals because of (c). Therefore, $\left\{P_{k}\right\}_{k=0}^{\infty}$ is the sequence of dyadic partitions $\left\{J_{k}\right\}_{k=0}^{\infty}$.

To complete the proof of Theorem 2, it suffices to prove the following assertion. If $\left\{f_{n}(x)\right\}_{n=0}^{2^{k-1}}$ is an orthonormal set of step functions $\left(J_{k}\right)$ satisfying (c) such that $\left|f_{n}(x)\right| \equiv 1,0 \leqq n \leqq 2^{k}-1$, then the given set is the set of Walsh functions $\left\{\psi_{n}(x)\right\}_{n=0}^{2^{k-1}}$ (in some order).

The proof is by induction. When $k=0$, the assumptions imply $f_{0}(x) \equiv 1$ $\equiv \psi_{0}(x)$ and the assertion is true. Assuming it true for $k$, consider a set $\left\{f_{n}(x)\right\}_{n=0}^{2^{k+1}-1}$ satisfying the given conditions.

Let $2^{k} \leqq n \leqq 2^{k+1}-1$. We claim that on two successive intervals of the form $I(2 r, k+1)$ and $I(2 r+1, k+1), f_{n}(x)$ takes values $\epsilon$ and $-\epsilon$ respectively $(\epsilon= \pm 1)$. To see this let $\chi(x)$ be the characteristic function of $I(r, k)$ $=I(2 r, k+1) \cup I(2 r+1, k+1)$. Since $\left\{f_{j}(x)\right\}_{j=0}^{2^{k-1}}$ is clearly a basis for the space of step functions $\left(J_{k}\right)$,

(2) For particulars on the Walsh functions see [1]. 


$$
\chi(x)=\sum_{j=0}^{2^{k}-1} a_{j} f_{j}(x)
$$

for appropriate coefficients $a_{j}$. Suppose $f_{n}(x)$ takes the values $\epsilon$ and $\boldsymbol{\epsilon}^{\prime}$ on $I(2 r, k+1)$ and $I(2 r+1, k+1)$ respectively. Then by orthogonality,

$$
0=\sum_{j=0}^{2^{k}-1} a_{j} \int_{I} f_{j}(x) f_{n}(x) d x=\int_{I} \chi(x) f_{n}(x) d x=\int_{I(r, k)} f_{n}(x) d x=\frac{\epsilon+\epsilon^{\prime}}{2^{k+1}} .
$$

Hence $\boldsymbol{\epsilon}^{\prime}=-\boldsymbol{\epsilon}$.

The above property is also possessed by the Rademacher function $\phi_{k}(x)$ defined by $\phi_{k}(x) \equiv(-1)^{r}$ on $I(r, k+1)$. Thus while $f_{n}(x)$ is a step function $\left(P_{k+1}\right)$, the product $\phi_{k}(x) f_{n}(x)$ is a step function $\left(P_{k}\right)$.

Consider the functions $\left\{g_{n^{\prime}}(x)=\phi_{k}(x) f_{2^{k}+n^{\prime}}(x)\right\}_{n^{\prime}=0}^{2^{k}-1}$. We claim they satisfy all the conditions of our assertion. Since $\left|\phi_{k}(x)\right| \equiv 1$, it is clear that $\left|g_{n^{\prime}}(x)\right| \equiv 1$ and that the $g_{n^{\prime}}(x)$ form an orthonormal set of step functions $\left(P_{k}\right)$. It remains to show that (c) holds.

Let $n=2^{k}+n^{\prime}$ where $0 \leqq n^{\prime} \leqq 2^{k}-1$. From our assumptions $f_{n}(x)$ has $n+1$ intervals of constancy, or equivalently, $n$ discontinuities. The latter occur among the dyadic rationals $r \cdot 2^{-(k+1)}, 1 \leqq r \leqq 2^{k+1}-1$. Multiplication by $\phi_{k}(x)$ removes these discontinuities (since $\left|f_{n}(x)\right| \equiv 1$ ), but introduces new ones at the remaining dyadic rationals $r \cdot 2^{-(k+1)}$. Therefore, $g_{n^{\prime}}(x)=\phi_{k}(x) f_{n}(x)$ has exactly $2^{k+1}-1-n=2^{k}-1-n^{\prime}$ jumps. Hence, the set $\left\{g_{n^{\prime}}(x)\right\}_{n^{\prime}=0}^{2^{k}-1}$ can be reordered so that (c) is satisfied (set $h_{n^{\prime}}(x)=g_{2}{ }^{k}-1-n^{\prime}(x)$ ). By the induction hypothesis, this set as well as $\left\{f_{j}(x)\right\}_{j=0}^{2^{k-1}}$ is the set $\left\{\psi_{j}(x)\right\}_{j=0}^{2^{k}-1}$.

Since $\phi_{\boldsymbol{k}}^{-1}(x)=\phi_{k}(x), f_{n}(x)=\phi_{k}(x) g_{n^{\prime}}(x)$. Thus, the set $\left\{f_{j}(x)\right\}_{j=2^{k}}^{2^{k+1}-1}$ is obtained when the Walsh functions $\left\{\psi_{j}(x)\right\}_{j=0}^{2^{k}-1}$ are multiplied by $\phi_{k}(x)$. But this is precisely the definition of the Walsh functions $\left\{\psi_{j}(x)\right\}_{j=2^{k}}^{2^{k+1}-1}$. Therefore $\left\{f_{j}(x)\right\}_{j=0}^{2^{k+1}-1}$ is the set of Walsh functions $\left\{\psi_{j}(x)\right\}_{j=0}^{2^{k+1}-1}$ (in some order) which completes the induction.

\section{REFERENCES}

1. N. J. Fine, On the Walsh functions, Trans. Amer. Math. Soc. vol. 65 (1949) pp. 372-414.

2. J. J. Price, Orthonormal sets with non-negative Dirichlet kernels, Trans. Amer. Math. Soc. vol. 95 (1960) pp. 256-262.

\section{CORNELl UNIVERSITY,} ITHACA, NEW YoRK 